\title{
Latent heat storage by silica-coated polymer beads containing organic phase change materials
}

Tivadar Feczkóa, ${ }^{\mathrm{a}, \mathrm{b}^{*}}$, László Trif ${ }^{\mathrm{a}}$, Daniel Horák ${ }^{\mathrm{c}}$

${ }^{a}$ Institute of Materials and Environmental Chemistry, Research Centre for Natural Sciences, Hungarian Academy of Sciences, Magyar tudósok körútja 2., 1117, Budapest, Hungary

${ }^{b}$ Research Institute of Chemical and Process Engineering, Faculty of Information Technology, University of Pannonia, Egyetem u. 10, H-8200 Veszprém, Hungary

${ }^{c}$ Institute of Macromolecular Chemistry, Academy of Sciences of the Czech Republic, Heyrovsky Sq. 2, 16206 Prague 6, Czech Republic

*E-mail: tivadar.feczko@gmail.com, phone: +36-88-623508, fax: +36-88-624038

\begin{abstract}
Phase change materials (PCMs) are used for latent heat storage. Most promising applications of PCMs are waste heat recovery systems, solar heating systems, building energy conservation systems, and air-conditioning systems. Macroporous poly(ethylene dimethacrylate) (PEDMA) carrier beads were formed by the suspension polymerization technique, imbibed with paraffin or cetyl alcohol phase change materials and coated with a silica shell to prevent their aggregation and the leakage of PCM. PEDMA beads contained $75.6 \%$ paraffin and $53.1 \%$ cetyl alcohol resulting in high latent heat storage capacities. The melting and crystallizing enthalpies were 132.6 and $133.4 \mathrm{~J} / \mathrm{g}$, respectively for paraffin-loaded capsules, while 89.6 and $88.6 \mathrm{~J} / \mathrm{g}$, respectively for cetyl alcohol containing beads. The excellent latent heat storage of the capsules was retained after 1,000 heating-cooling cycles indicating that the capillary forces of the silicacoated porous polymer beads efficiently prevented the leakage of the PCMs.
\end{abstract}

Keywords: latent heat storage, phase change materials, porous beads by suspension polymerization, silica shell. 


\section{Introduction}

A reduction of the energy consumed in buildings is one of the priorities of the EU aims. To achieve this goal it is essential to enlarge the share of renewable and natural energy sources for the heating, ventilation and cooling of buildings (Arkar and Medved, 2007). Solar energy is considered to be one of the most promising sources of energy, owing to its clean and renewable characteristics ( $\mathrm{Wu}$ and $\mathrm{Zhao}, 2011$ ). The use of solar energy for heating has been utilized for a long time in passive systems; however, the efficiency of natural heating and cooling could be enhanced by equilibrating the daily fluctuations. Thermal energy storage provides a reservoir of energy to adjust the gap between the demand and the availability. Latent heat storage is one of the most attractive techniques due to high storage density. Phase change materials (PCMs) can store a large amount of thermal energy with a constant temperature due to their high latent heat during the phase transition. However, the applications of PCMs in the thermal energy storage systems are limited by their low thermal conductivities. The major drawback of latent thermal energy storage can be diminished substantially by microencapsulation. Recently, microencapsulation of PCMs has been utilized in thermal energy storage, functional fibers, thermal insulation, heating, and air conditioning of buildings (Sari et al., 2009). Moreover, a PCM-based storage unit has much less weight and after optimal engineering the energy stored by conventional building materials could be improved (Saman and Halawa, 2005).

If the microcapsules are dispersed in a fluid (mostly water), a pumpable slurry is formed, that can be used as an energy transport- and storage-medium, a so-called PCM slurry. PCM is present as the core in microcapsules and a polymer or inorganic shell maintains the shape and prevents PCM leakage during the phase change process (Qiu et al., 2013). The advantages of microencapsulated PCMs are the protection of PCM against the influences of the environment, increasing the specific heat-transfer area, and due to the suitable coating the composite withstands changes in the volume of PCM, as the phase change occurs (Li et al., 2011). Significant efforts have been made to improve the thermal conductivity of PCMs by shapestabilization and microencapsulation. An effective mechanism can be the incorporation of metal of high thermal conductivity, e.g., $\mathrm{Cu}$ ions were successfully utilized for this purpose (Tang et al., 2012).

Paraffins are versatile PCMs, since one can select an appropriate compound from a wide melting and solidifying temperature range for the required application. They have large latent heat 
capacity, low vapour pressure, negligible supercooling, good thermal and chemical stability, and self-nucleating behaviour. Cetyl alcohol is also a useful PCM possessing the mentioned beneficial properties. Most recently, Tang et al. (2016) synthesized cetyl alcohol-dyepolyurethane composite by entrapping the PCM into a visible light-driven matrix. The novel composite presented an extremely high phase-change enthalpy and suitable phase change temperature.

Several methods have been used for microencapsulation of paraffins by entrapping them into organic shells by e.g. emulsion-solvent evaporation (Feczkó et al., 2014) or coacervation (Uddin et al., 2002). Miniemulsion method (Zhang et al., 2012) and in situ polymerization of organic shell are also frequently used for the production of capsules composed of PCM core and polymer shell (Ma et al., 2012). Other techniques include absorption, solution intercalation, and ultrasound assisted sol-gel method (Tang et al., 2014) that offers advantage of simplicity and low cost (Tang et al., 2013). Optionally, liquid PCM is mixed with a molten polymer to create a supporting matrix similar to a foam within the PCM itself. These types of composites are generally entitled as shape-stable PCMs. The polymer matrix provides a compact shape to the PCM, and when it melts, the liquid phase is fully contained within the matrix due to capillary forces (Ehid et al 2012). Since the energy storage is the function of the amount of PCM, it is desirable to stabilize the material with as little amount of polymer as possible. The shape-stable PCMs can be classified into two groups: solid-solid and solid-liquid PCMs (Hong and Ge, 2000). The first group involves the molecular crystals, which undergo solid-state crystal transformations accompanied with absorbing/releasing sufficient heat, and polymers cross-linked by chemical agents or by electron beam irradiation. The second category includes composites obtained by dispersing PCMs into higher melting-point materials acting as supporting media. As long as the operating temperature is below the melting point of the supporting material, the composite preserves its shape even if the PCM changes from solid to liquid.

In this study, a novel approach was utilized to form new type of PCM composite capsules. Two generally applied techniques were combined for the first time according to our knowledge, in order to improve the properties of the formed PCM capsules. That is, shape-stable PCMs were covered by silica shell prepared by sol-gel method. Shape-stabilization was achieved by preparing porous poly(ethylene dimethacrylate) (PEDMA) bead carrier using suspension radical polymerization, and by filling them with paraffin or cetyl alcohol PCMs via dipping. To inhibit 
PCM leaking, the silica shell was prepared by hydrolysis and condensation of silane. The PEDMA microspheres have the function of shape-stabilized PCM, which prevents leakage. As shown in another work (Khadira et al., 2015), the porous network may prevent the leakage of PCM by the capillary and surface tension forces. Additionally, the layer of silica nanoparticles is capable to inhibit the PCM leakage. This complex composite is more advantageous compared to the simple core-shell structured microcapsules, since our microspheres would presumably keep the most of the PCM even after a surface damage.

\section{Experimental}

\subsection{Materials}

Ethylene dimethacrylate (EDMA; Ugilor S.A., France) was distilled before use under vacuum. 2,2'-Azobis(isobutyronitrile) (AIBN; recrystallized from ethanol), [(2hydroxypropyl)methyl]cellulose (Methocel $90 \mathrm{HG}$ ), cetyl alcohol (hexadecane-1-ol, CA), and dibenzoyl peroxide (BPO) were obtained from Fluka (Buchs, Switzerland). Trimethoxy(methyl)silane (TMMS) and Tween 20 (polyoxyethylene (20) sorbitan monolaurate) were from Sigma-Aldrich and the paraffin mixture $(\mathrm{P})$, melting-solidification range $53-55{ }^{\circ} \mathrm{C}$, from Paramo (Pardubice, Czech Republic). Other chemicals and solvents were from Lachner (Neratovice, Czech Republic).

\subsection{Synthesis of macroporous beads}

The PEDMA beads, later on filled with cetyl alcohol, were prepared via polymerization of EDMA as follows. Aqueous 0.2 wt.\% Methocel $90 \mathrm{HG}$ solution (6 1) was charged in a 101 reaction vessel, solution of EDMA $(800 \mathrm{~g})$, toluene $(885 \mathrm{~g}), 1,2$-dichloroethane $(315 \mathrm{~g})$ and AIBN ( $4 \mathrm{~g})$ were added under stirring at $90 \mathrm{rpm}$, and the reaction mixture was heated to $75^{\circ} \mathrm{C}$ for $6 \mathrm{~h}$. The resulting beads were washed with water, toluene and methanol, and dried at room temperature.

The PEDMA beads, later on filled with paraffin, were obtained by polymerizing EDMA (7.5 or 6 g) with BPO (0.075 or $0.06 \mathrm{~g})$ and cyclohexyl acetate $(22.5$ or $24 \mathrm{~g})$ in aqueous $0.1 \mathrm{wt} . \%$ Methocel $90 \mathrm{HG}$ solution $(90 \mathrm{ml})$ at $70{ }^{\circ} \mathrm{C}$ for $16 \mathrm{~h}$ under stirring at $300 \mathrm{rpm}$ under $\mathrm{CO}_{2}$ atmosphere. The resulting beads were washed with water, acetone, water, and dried at room 
temperature and then at $80^{\circ} \mathrm{C}$. The two types of beads described above were prepared in reaction vessels of different sizes. Large reaction vessel requires lower stirring speed than the small one, if stirring intensity is intended to be the same, i.e., to get particles of approximately similar size.

\subsection{Loading of phase change materials into beads and encapsulation by silica shell}

Cetyl alcohol and paraffin were selected as phase change materials to be incorporated, because they possess high latent heat. Macroporous PEDMA beads (2 g) were dispersed in molten PCM $(5 \mathrm{~g})$ which was loaded at $65-100{ }^{\circ} \mathrm{C}$. The particles were separated by centrifugation using a stainless sieve and again dispersed in aqueous $0.1 \mathrm{wt} . \%$ Methocel $90 \mathrm{HG}$ solution $(20 \mathrm{ml})$ at 70 ${ }^{\circ} \mathrm{C}$ in 50-ml reaction vessel under stirring with an anchor-type stirrer (500 rpm).

TMMS (1.36 g; $10 \mathrm{mmol})$ was hydrolyzed in $0.01 \mathrm{M} \mathrm{HCl}(0.54 \mathrm{~g} ; 30 \mathrm{mmol})$ at room temperature to yield $\mathrm{SiMe}(\mathrm{OR})_{3}$ hydrolysate. The volume of the mixture was adjusted to $5 \mathrm{ml}$ by adding water. The TMMS hydrolysate $(1 \mathrm{ml})$ was added to the resulting PCM-PEDMA beads, the suspension was stirred at $70{ }^{\circ} \mathrm{C}$ for $3.5 \mathrm{~h}$, and the product was washed eight times in $0.01 \mathrm{wt} . \%$ Tween 20 (100 $\mathrm{ml}$ each). Finally, the composite beads were dried in air. The scheme of macroporous beads imbibing and coating by silica is shown in Figure 1.

\subsection{Characterization methods}

Carl Zeiss optical microscope (Germany) and Philips/FEI XL30 environmental scanning electron microscope (ESEM) operated at $25 \mathrm{kV}$ were used for the imaging of the particle surface. The surface was sputter-coated by a $15-\mathrm{nm}$ thick $\mathrm{Au} / \mathrm{Pd}$ layer before the SEM investigation. Silica shell was analysed using the same electron microscope equipped with EDAX Genesis EDS spectrometer operated upon energy dispersive X-ray spectroscopy. Quanta 200 FEG scanning electron microscope (SEM; Fei, Brno, Czech Republic) was used for the observation of the particle cross-sections; the particles were embedded in London White Resin (LWR) and ultramicrotomed (Ultrotome III, LKB BROMMA 8800). The particle size distribution was analysed by the laser diffraction method (Mastersizer 2000, Malvern Instruments, UK). To have homogeneous dispersion during size analysis, the Malvern Hydro 2000SM dispersion unit was used at a speed of $1500 \mathrm{rpm}$. The average particle sizes were expressed in equivalent volume mean diameters (D [4.3]). 
The PCM content was calculated after the beads $(100 \mathrm{mg})$ were crushed in a mortar, and the PCM was extracted 3 times from the particles by chloroform $(5 \mathrm{ml}$ each). The remaining material was weighed and the PCM content calculated. The specific surface area $\left(S_{\mathrm{BET}}\right)$ of the microspheres was determined by nitrogen adsorption (77 K) using a Gemini VII 2390 Analyser (Micromeritics; Norcross, GA, USA). Pore volume was determined from cyclohexane or 1chlorododecane regain using centrifugation method (Stamberg and Sevcik, 1966).

Thermogravimetric analyses (TGA) were performed in air $(50 \mathrm{ml} / \mathrm{min})$ using a Perkin Elmer Pyris 1 Thermogravimetric Analyser over a temperature interval $30-600{ }^{\circ} \mathrm{C}$ at a heating rate of 10 ${ }^{\circ} \mathrm{C} \min ^{-1}$.

The thermal properties of the microcapsules were determined using a Setaram $\mu$ DSC3evo differential scanning microcalorimeter. The beads were weighed into $100 \mu l$ aluminium crucibles, and ethylene glycol as a heat transferring medium was added, which covered the particles, and the system was closed by a cap. The samples were cycled with a scanning rate of $0.6^{\circ} \mathrm{C} / \mathrm{min}$. The results were processed by the thermoanalyzer's Calisto Processing (v1.38) software. The corresponding melting/freezing enthalpies and onset temperatures were determined by the baseline integration method (Tangential sigmoid baseline type).

In thermal cycling tests the thermal reliability of the microencapsulated PCMs was determined. One thermal cycling test included the heating till the complete melting by exceeding the melting points with $20{ }^{\circ} \mathrm{C}$, and the cooling to $20^{\circ} \mathrm{C}$ below the freezing points, resulting in the complete freezing of the phase change materials in the capsules. One heating-cooling cycle took 4 minutes. The tests were performed consecutively up to 1,000 thermal cycles were performed. The microcapsules were warmed by a heating circulator (Julabo EH, Germany) and cooled by a Peltier element. DSC analysis was performed using Setaram $\mu \mathrm{DSC} 3$ evo in order to investigate the thermal reliability of the composite PCMs after thermal cycling.

\section{Results and Discussion}

\subsection{Size, morphology, surface area, porosity and PCM content in the beads}

Macroporous beads were prepared by the AIBN/BPO-initiated suspension radical polymerization of EDMA in the presence of an inert porogen. Based on preliminary polymerizations, the porogen for the preparation of cetyl alcohol-containing PEDMA beads was a mixture of toluene 
and 1,2-dichloroethane. The polymerization of neat EDMA provided highly crosslinked particles possessing high specific surface area and small pores (Table 1). PEDMA beads intended for cetyl alcohol absorbtion were 0.5-1.5 $\mathrm{mm}$ in size (average diameter $758 \mu \mathrm{m}$ ) (Table 2, Figure 2), had specific surface area $435 \mathrm{~m}^{2} / \mathrm{g}$, and pore volume $3 \mathrm{ml} / \mathrm{g}$.

PEDMA microspheres for paraffin uptake were prepared using cyclohexyl acetate porogen. Their particle size distribution was broader $(0.6-2 \mathrm{~mm})$ than that of the beads prepared for cetyl alcohol imbibing. The specific surface area of beads was $513 \mathrm{~m}^{2} / \mathrm{g}$ (Table 1). As expected, the pore volume of these microspheres was substantially larger $(3.9 \mathrm{ml} / \mathrm{g})$ than that of the carrier of cetyl alcohol. Both types of the carrier beads had spherical shape (Figure 3). The higher polydispersity was found in beads filled with paraffin, analyzed by optical microscopy. The SEM of crosssection of beads embedded in LWR resin is shown in Figure 4. While the PEDMA particles synthesized for cetyl alcohol absorbtion contained numerous macropores (Figure 4A), the inner morphology of the PEDMA beads for paraffin imbibing (Figure 4B) contained smaller and rather homogeneous pores.

It is an important advantage of the PEDMA beads that they are rather hydrophobic, i.e., compatible with the PCMs, which facilitated their fast imbibition in the pores and air displacement thanks to the low interfacial tension. After PCM loading, the particles were dispersed in aqueous [(2-hydroxypropyl)methyl]cellulose solution and coated with a silica shell using trimethoxy(methyl)silane (TMMS) hydrolysate. It was shown in a previous report that condensation of silanols results in siloxanes which interact with the particle surface (Grama et al., 2014).

The hydrolysate was formed from TMMS under mild conditions, i.e., in highly diluted $\mathrm{HCl}$ solution at room temperature. Finally, the beads were thoroughly washed to remove nonencapsulated PCM. The pores of PEDMA beads were fully filled with the amorphous PCM (Figure 5A). Using [(2-hydroxypropyl)methyl]cellulose as a stabilizer, individual and separated silica-coated polymer beads were formed and well-dispersed in water. Compact silica shell composed of nanoparticles on the surface of the polymer microspheres was confirmed by the SEM image (Figure 5B); moreover, presence of the silica was confirmed by EDAX analysis (Figure 5C).

\subsection{Thermal stability}


Appropriate thermal stability is a necessary and important property of engineered PCMs in thermal energy storage and temperature regulation, therefore, thermogravimetric analysis is frequently used to assess the thermal resistance of PCMs. The cetyl alcohol and the paraffin evaporated at $200-300{ }^{\circ} \mathrm{C}$ and $300-400{ }^{\circ} \mathrm{C}$, respectively (Figure 6). The PEDMA decomposed mostly in the temperature range $220-550{ }^{\circ} \mathrm{C}$, since as indicated in a recent work (Grama et al., 2014) the formation of silica shell on the microspheres retarded the thermo-oxidative degradation of the organic carrier, most likely because the silica shell limited the amount of air accessible to the organic material. This result suggests that the shape-stabilized PCM has a good heat resistant performance, consequently a broad applicable temperature range.

\subsection{DSC analysis}

To investigate the phase change properties of engineered PCMs, DSC analysis is the most useful technique. The DSC measurements of the composites were performed in the presence of ethylene glycol heat transferring medium. Figure 7 shows the DSC curves of PEDMA-paraffin and PEDMA-cetyl alcohol composites coated by silica, and DSC from the pure PCMs are also displayed together with the DSCs before and after the cycling. The enthalpy change corresponding to the lower temperature is the result of the solid-solid phase transition of the PCMs. The melting and freezing temperature of paraffin showed negligible variations in the composites. However, these temperatures of cetyl alcohol decreased significantly after shapestabilization and incorporating in the silica shell.

The melting and crystallizing enthalpies of the pure paraffin were 203.2 and $198.7 \mathrm{~J} / \mathrm{g}$, respectively (Figure 7A), while these values for PEDMA-paraffin beads were 132.6 and 133.4 $\mathrm{J} / \mathrm{g}$, respectively (Figure 7C), which were somewhat lower values than those calculated from their paraffin content $(75.6 \%)$. This difference can be explained by the thermal isolating effect of the silica shell. The melting and solidifying latent heats of cetyl alcohol were 204.3 and $218 \mathrm{~J} / \mathrm{g}$, respectively (Figure 7B). After its incorporation in the silica-coated PEDMA beads, these values decreased to 89.6 and $88.6 \mathrm{~J} / \mathrm{g}$ (Figure 7D), which are also lower than those calculated from the PCM content (53.1\%). The reduction is interpreted again by the presence of the silica shell, since its thermal conductivity is lower than that of the PCM and creates a thermal resistance barrier between the heat source and the PCM itself. 
The multiple peaks on the heating and cooling curves of the fillers and PCM beads are the result of various solid-solid and solid-liquid phase transitions. In the Figures 7A and 7C, respectively (pure paraffin and PEDMA-paraffin beads), two peaks can be identified on both the heating and cooling curve. The first, smaller endotherm is a solid-solid transition of paraffin, when the orthorhombic or monoclinic (depending on the number of carbon atoms in the molecule) crystalline structure transforms into close-packed hexagonal system. This phenomenon is profoundly described in the literature (Craig et al., 1967, Ukrainczyk et al., 2010). The second, larger endotherm corresponds to the solid-liquid transition, i.e., the melting point of the paraffin. Obviously, during cooling the order is reversed, that is, the first event is the liquid-solid (crystallization) transition, while at lower temperatures the solid-solid transition takes place.

The fatty alcohols are also showing polymorphism, similar to the paraffin described above. The polymorphs stable at room temperature are $\gamma$ and $\beta$ (monoclinic phase), while the $\alpha$ form (hexagonal phase) which is stable at higher temperatures, melts at room temperature. During heating, the solid-solid transitions ( $\gamma$ and $\beta$ to $\alpha$ transitions, which are first order transitions) are overlapping with the solid-liquid transformation (melting of the form $\alpha$ ), and thus these two processes appear as a single endothermic peak (see the heating curve on the Figure 7B). During cooling, the above mentioned two processes separate from each other, that's why two exothermic peaks are distinguishable, that is, solidification of the liquid phase, forming $\alpha$ phase, and at lower temperatures transition of the $\alpha$ - to $\gamma$-phase (Ventola et al, 2002, Ribeiro et al., 2004, Katdare et al., 2006).

Regarding the applicability of PCM-containing microcapsules, the absence of latent heat storage material leakage is essential during the phase change. Thus, the thermal test including 1,000 heating/cooling cycles involving complete melting/freezing of PCM was investigated. The mass of particles did not change after the thermal cycling, furthermore, their heat capacity was investigated by DSC again. Both crystallizing and melting enthalpies of paraffin (Figure 8A) and cetyl alcohol (Figure $8 \mathrm{~B}$ ) containing composites indicated negligible decrease related to the corresponding values before 1,000 heating-cooling cycles (PEDMA-paraffin-silica: Figure 7C, PEDMA-cetyl alcohol-silica: Figure 7D). Nevertheless, it should be emphasized that this quick thermal cycling test cannot model the reliability of the microspheres in real service. In order to get reliable information on their long-term performance, investigations under stress conditions simulating their real use would be desirable. 


\section{Conclusions}

A novel approach was used to microencapsulate paraffin and cetyl alcohol phase change materials in the macroporous methacrylate-based beads prepared by the suspension polymerization method, and the PCMs were filled into the PEDMA carriers by imbibing. In order to prevent particle adhesion/aggregation and PCM release, the PCM-loaded beads were coated with a protective silica shell composed of silica nanoparticles. Both composites possessed high heat capacity, nevertheless, due to the substantially higher PCM content the PEDMA-paraffin beads had significantly higher latent heat storage capacity. After 1,000 heating-cooling cycles it was verified that there was no leakage of the PCMs from the composites, which indicated that the composites had excellent shape-stable performances. Hence, it can be concluded that the capillary forces of the porous polymer carrier and the silica layer prevented the leakage of the PCMs.

\section{Acknowledgement}

We acknowledge the support of the Hungarian state and the European Union TAMOP-4.2.2A11/1/KONV-2012-0072 and TAMOP-4.1.1C-12/1/KONV-2012-0017. This paper was also supported by the János Bolyai Research Scholarship of the Hungarian Academy of Sciences.

\section{References}

Arkar, C., Medved, S., 2007. Free cooling of a building using PCM heat storage integrated into the ventilation system. Sol. Energ. 81, 1078-1087.

Craig, R. G., Powers, J. M., Peyton, F. A., 1967. Differential thermal analysis of commercial and dental waxes, J. Dent. Res. 46, 1090-1097.

Ehid, R., Fleischer, A.S., 2012. Development and characterization of paraffin-based shape stabilized energy storage materials. Energy Convers. Manage. 53, 84-91.

Feczkó, T., Kardos, F.A., Németh, B., Trif, L., Gyenis, J., 2014. Microencapsulation of nhexadecane phase change material by ethyl cellulose polymer. Polym. Bull. 71, 32893304. 
Grama, S., Boiko, N., Bilyy, R., Klyuchivska, O., Antonyuk, V., Stoika, R., Horak, D., 2014. Novel fluorescent poly(glycidyl methacrylate) - silica microspheres, Europ. Polym. J. 56, 92-104.

Hong, Y., Ge, X.S., 2000. Preparation of polyethylene-paraffin compound as a form-stable solidliquid phase change material. Sol. Energy Mater. Sol. Cells 64, 37-44.

Katdare, A., Chaubal, M. V., 2006. Excipient Development for Pharmaceutical, Biotechnology, and Drug Delivery Systems, Informa Healthcare, Taylor and Francis Group. LLC., ISBN13: 978-0-8493-2706-3, pp 199-201.

Khadira, T., Hussein, M.Z., Zainal, Z., Rusli, R., 2015. Shape-stabilised n-octadecane/activated carbon nanocomposite phase change material for thermal energy storage. J. Taiwan Inst. Chem. Eng. 55, 189-197.

Li, M.G., Zhang, Y., Xu, Y.H., Zhang, D., 2011. Effect of different amounts of surfactant on characteristics of nanoencapsulated phase-change materials. Polym. Bull. 67, 541-552.

Ma, Y., Chu, X., Li, W., Tang, G., 2012. Preparation and characterization of poly(methyl methacrylate-co-divinylbenzene) microcapsules containing phase change temperature adjustable binary core materials. Sol. Energ. 86, 2056-2066.

Stamberg J., Sevcik, S., 1966. Chemical transformations of polymers. 111. Selective hydrolysis of a copolymer of diethylene glycol methacrylate and diethylene glycol dimethacrylate, Collect. Czechoslov. Chem. Commun. 31, 1009-1016.

Qiu, X., Song, G., Chu, X., Li, X., Tang, G., 2013. Microencapsulated n-alkane with p(n-butyl methacrylate-co-methacrylic acid) shell as phase change materials for thermal energy storage, Sol. Energ. 91, 212-220.

Ribeiro, H. M., Morais, J. A., Eccleston, G. M., 2004. Structure and rheology of semisolid o/w creams containing cetyl alcohol/non-ionic surfactant mixed emulsifier and different polymers, Int. J. Cosm. Sci. 26, 47-59.

Saman, W., Halawa, F.B.E., 2005. Thermal performance of PCM thermal storage unit for a roof integrated solar heating system. Sol. Energ. 78, 341-349.

Sari, A., Alkan, C., Karaipekli, A., Uzun, O., 2009. Microencapsulated n-octacosane as phase change material for thermal energy storage. Sol. Energ. 83, 1757-1763.

Tang, B., Cui, J., Wang, Y., Jia, C., Zhang, S., 2013. Facile synthesis and performances of PEG/SiO2 composite form-stable phase change materials. Sol. Energ. 87, 484-492. 
Tang, B., Qiu, M., Zhang, S., 2012. Thermal conductivity enhancement of PEG/SiO2 composite PCM by in situ Cu doping. Sol. Energy Mater. Sol. Cells. 105, 242-248.

Tang, B., Wang, L., Xu, Y., Xiu, J., Zhang, S., 2016. Hexadecanol/phase change polyurethane composite as form-stablephase change material for thermal energy storage. Sol. Energy Mater. Sol. Cells. 144, 1-6.

Uddin, M.S., Zhu, H.J., Hawlader, M.N.A., 2002. Effects of cyclic operation on the characteristics of a microencapsulated PCM storage material. Int. J. Sol. Energ. 22, 105 114.

Ukrainczyk, N., Kurajica, S., Sipusic, J., 2010. Thermophysical comparison of five commercial paraffinwaxes as latent heat storage materials, Chem. Biochem. Eng. Q. 24, 129-137.

Ventola, L., Ramirez, M., Calvet, T., Solans, X., Cuevas-Diarte, M. A., 2002. Polymorphism of N-Alkanols: 1-Heptadecanol, 1-Octadecanol, 1-Nonadecanol, and 1-Eicosanol, Chem. Mater. 14, 508-517.

Wu, Z.G., Zhao, C.Y., 2011. Experimental investigations of porous materials in high temperature thermal energy storage systems. Sol. Energ. 85, 1371-1380.

Zhang, G. H.; Bon, S. A. F.; Zhao, C. Y., 2012. Synthesis, characterization and thermal properties of novel nanoencapsulated phase change materials for thermal energy storage. Sol. Energ. 86, 1149-1154. 
Figure captions

Figure 1 Scheme of the PEDMA beads imbibing with phase change material and their coating by silica shell.

Figure 2 Size distribution of PEDMA beads containing cetyl alcohol (CA-PEDMA) and paraffin (P-PEDMA) phase change materials and covered by silica shell.

Figure 3 Optical micrographs of PEDMA beads containing cetyl alcohol (A) and paraffin (B) phase change materials and covered by silica shell.

Figure 4 Internal morphology of PEDMA beads for cetyl alcohol (A) and paraffin (B) imbibing.

Figure 5 Surface of PEDMA beads without (A) and with (B) silica nanoparticles (10000x magnification) as well as $\operatorname{EDAX}(\mathrm{C})$.

Figure 6 Thermogravimetric analysis (A: TG, B: DTG) of paraffin, cetyl alcohol, PEDMAcetyl alcohol-silica and PEDMA-paraffin-silica beads.

Figure 7 DSC curves of paraffin (A), cetyl alcohol (B), PEDMA-paraffin-silica (C) and PEDMA-cetyl alcohol-silica (D) beads.

Figure 8 DSC curves of PEDMA-paraffin-silica (A) and PEDMA-cetyl alcohol-silica (B) beads after 1000 heating-cooling cycles. 


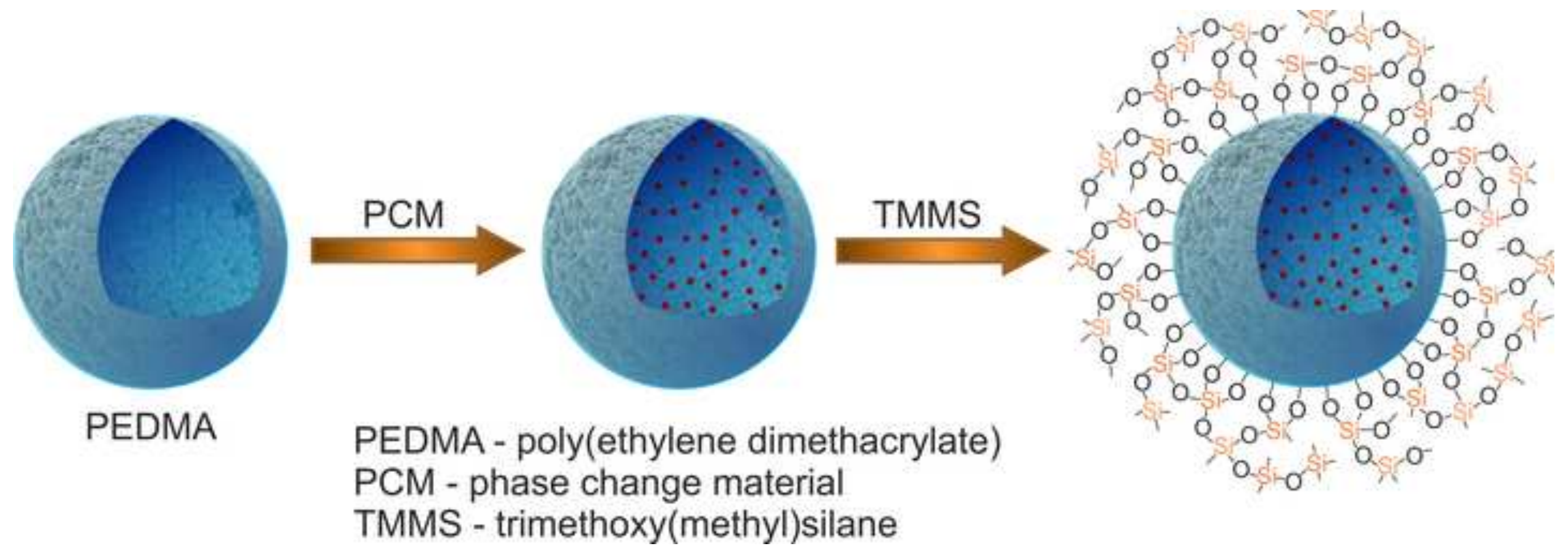




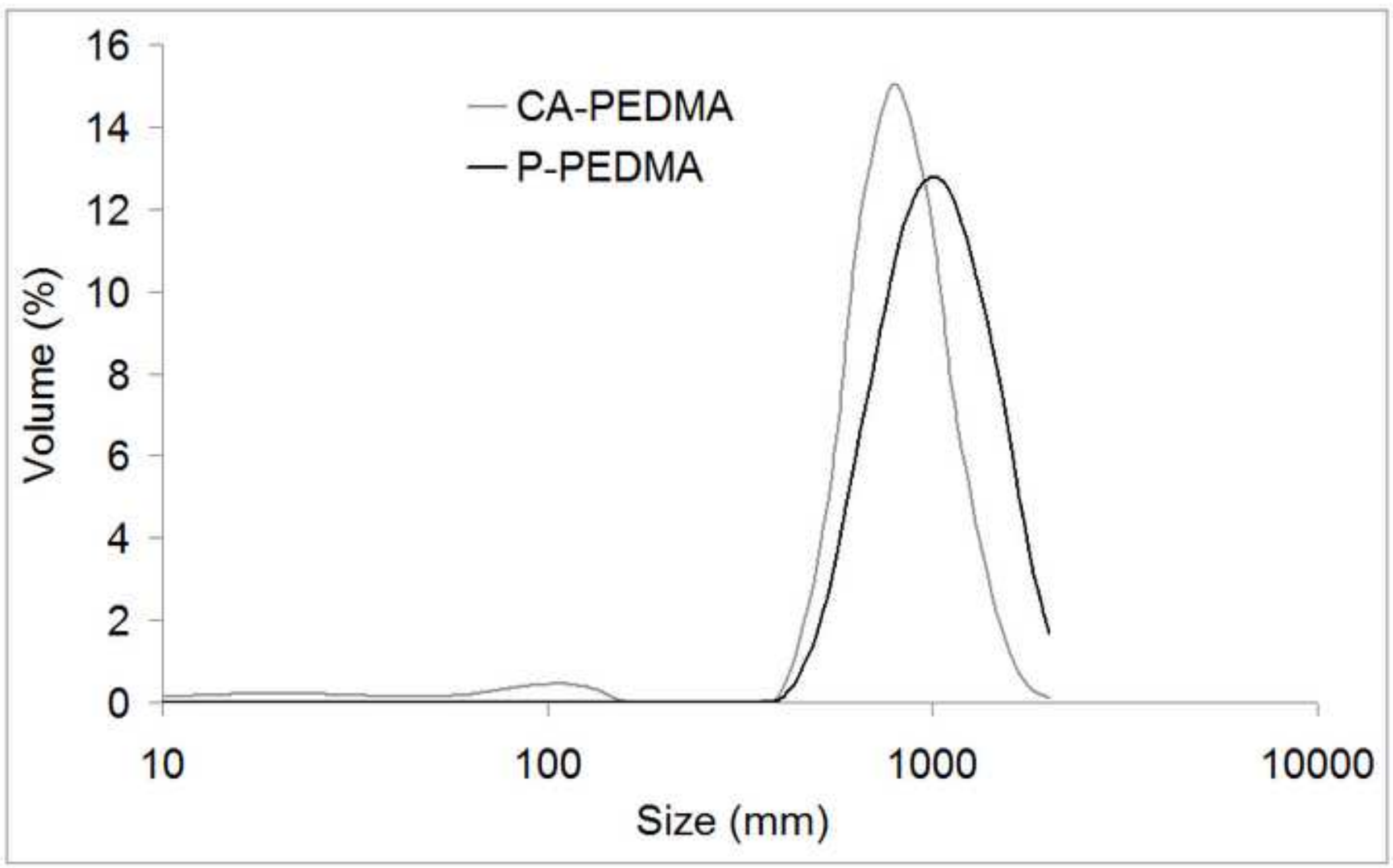




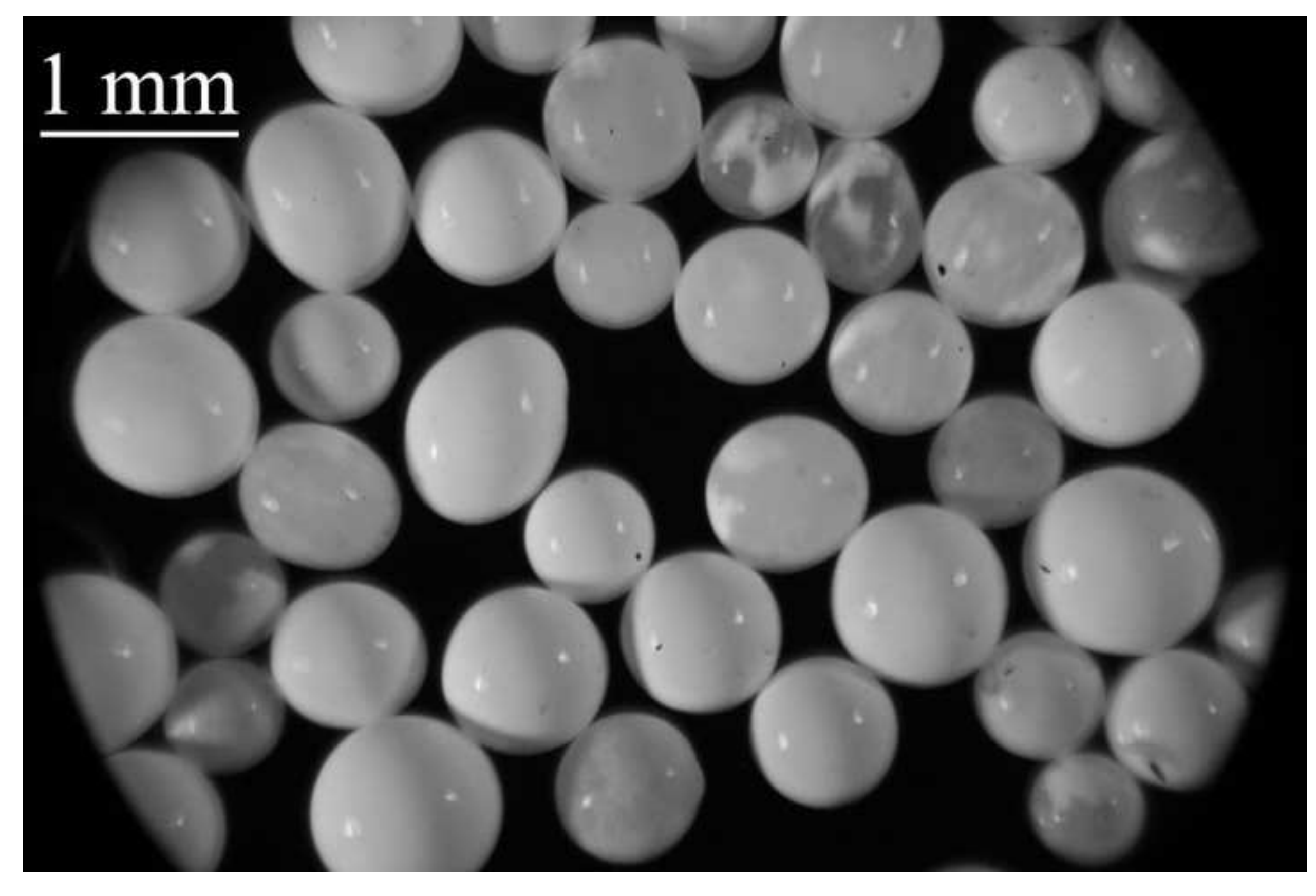

Clownload high resolution

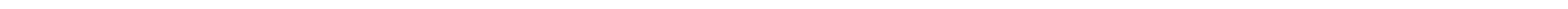




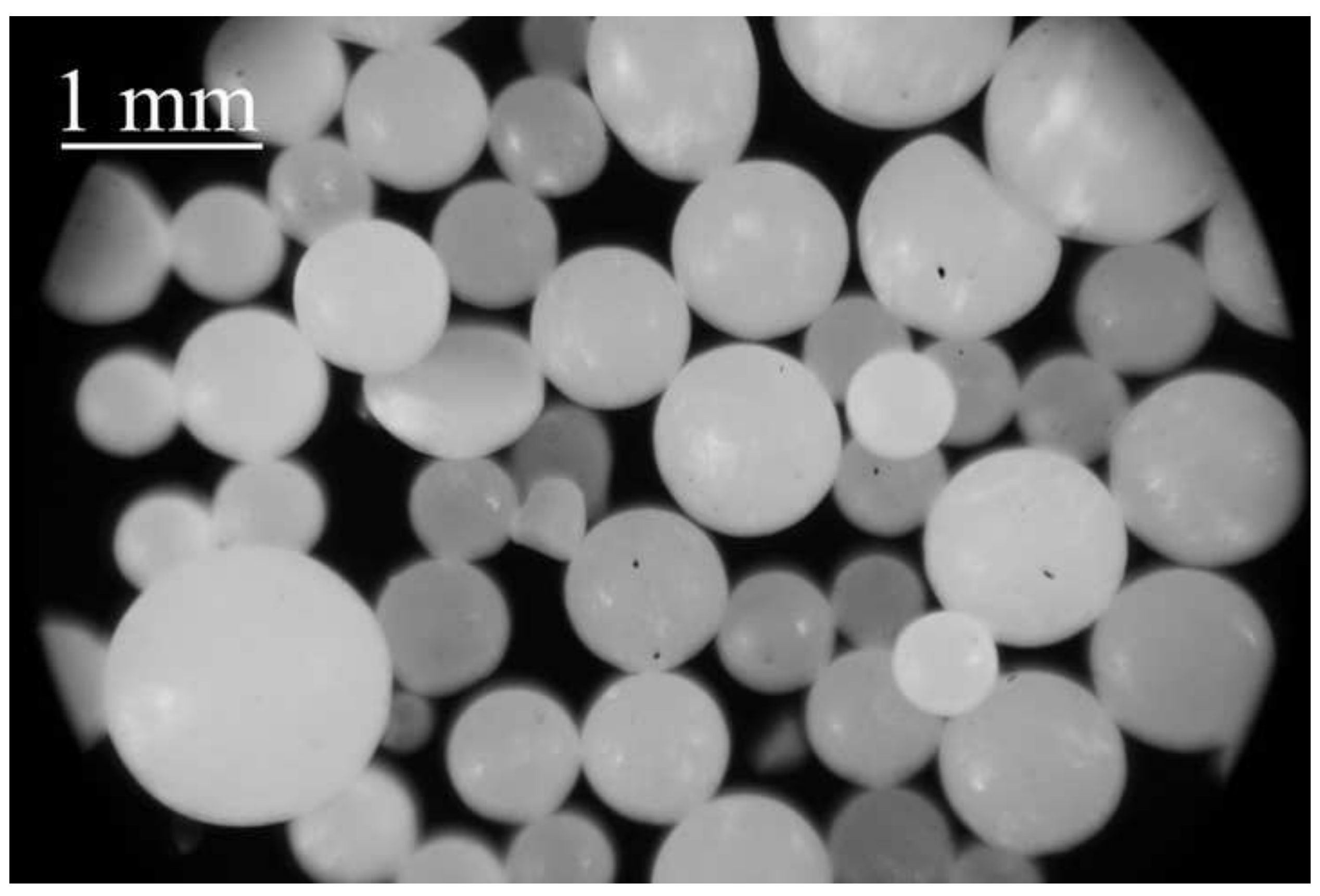

Click here to download high resolution image 


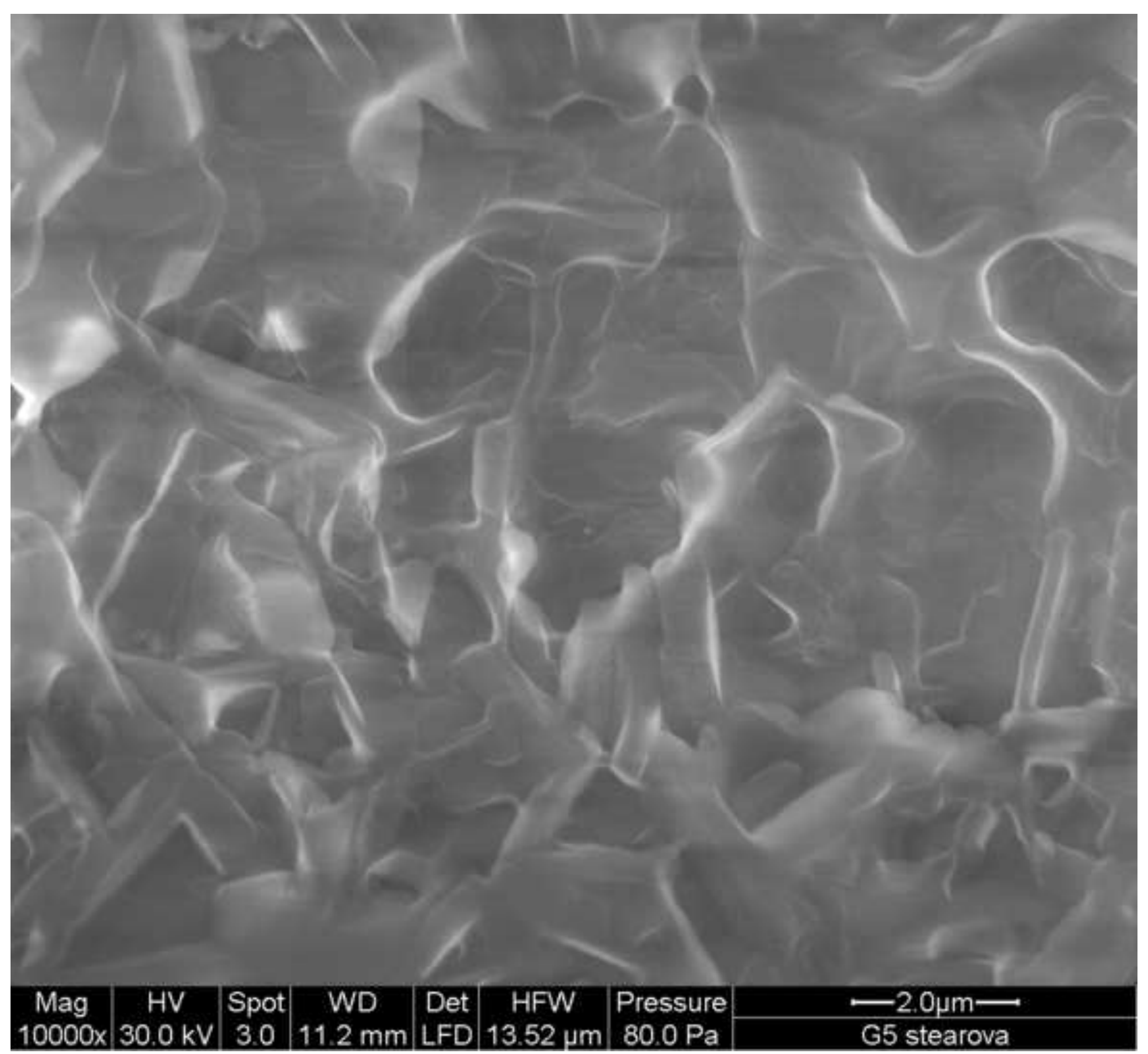

\begin{tabular}{c|c|c|c|c|c|c} 
Mag & HV & Spot & WD & Det & HFW & Pressure \\
$10000 \times$ & $30.0 \mathrm{kV}$ & 3.0 & $11.2 \mathrm{~mm}$ & LFD & $13.52 \mu \mathrm{m}$ & $80.0 \mathrm{~Pa}$ \\
\hline
\end{tabular}

G5 stearova 
Click here to download high resolution image
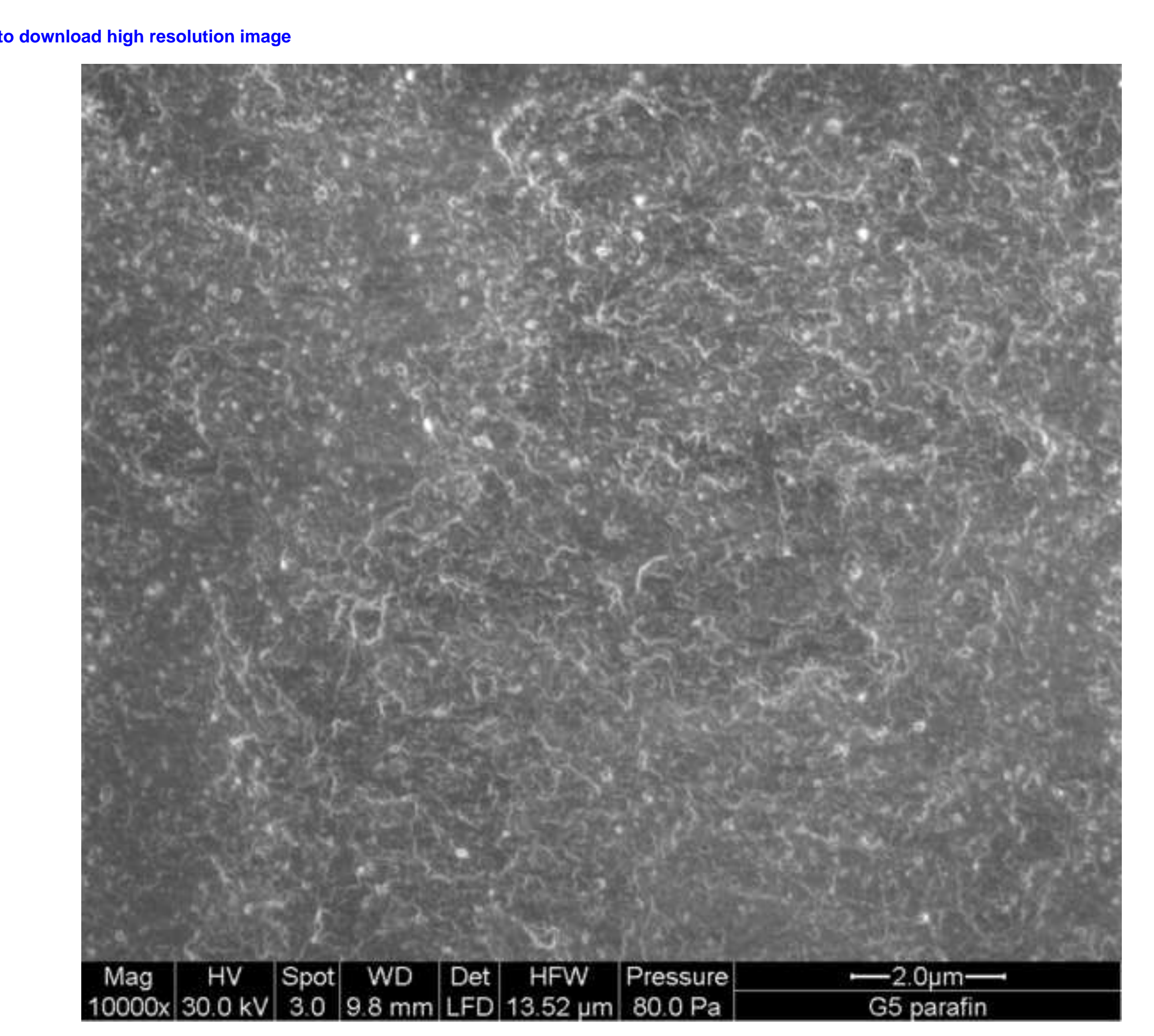

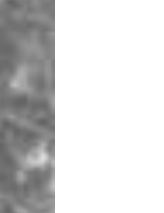$$
\text { . }
$$$$
\text { . }
$$

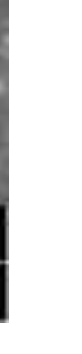




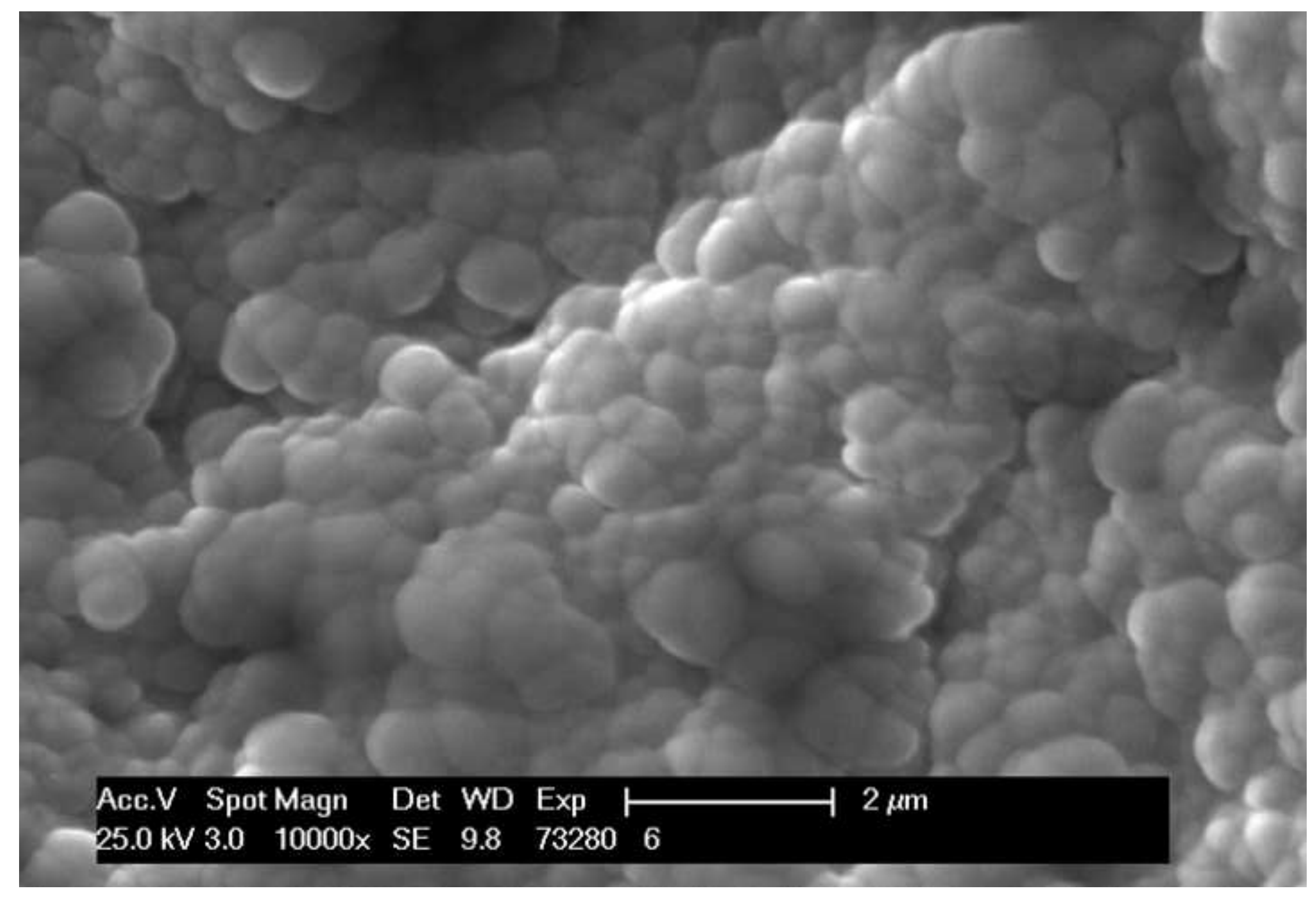

lick here to download high resolution image

$\begin{array}{lllllll}\text { Acc.V Spot Magn } & \text { Det WD } & \text { Exp } & & \\ 25.0 \mathrm{kV} & 3.0 \quad 10000 \times & \text { SE } & 9.8 & 73280 & \end{array}$




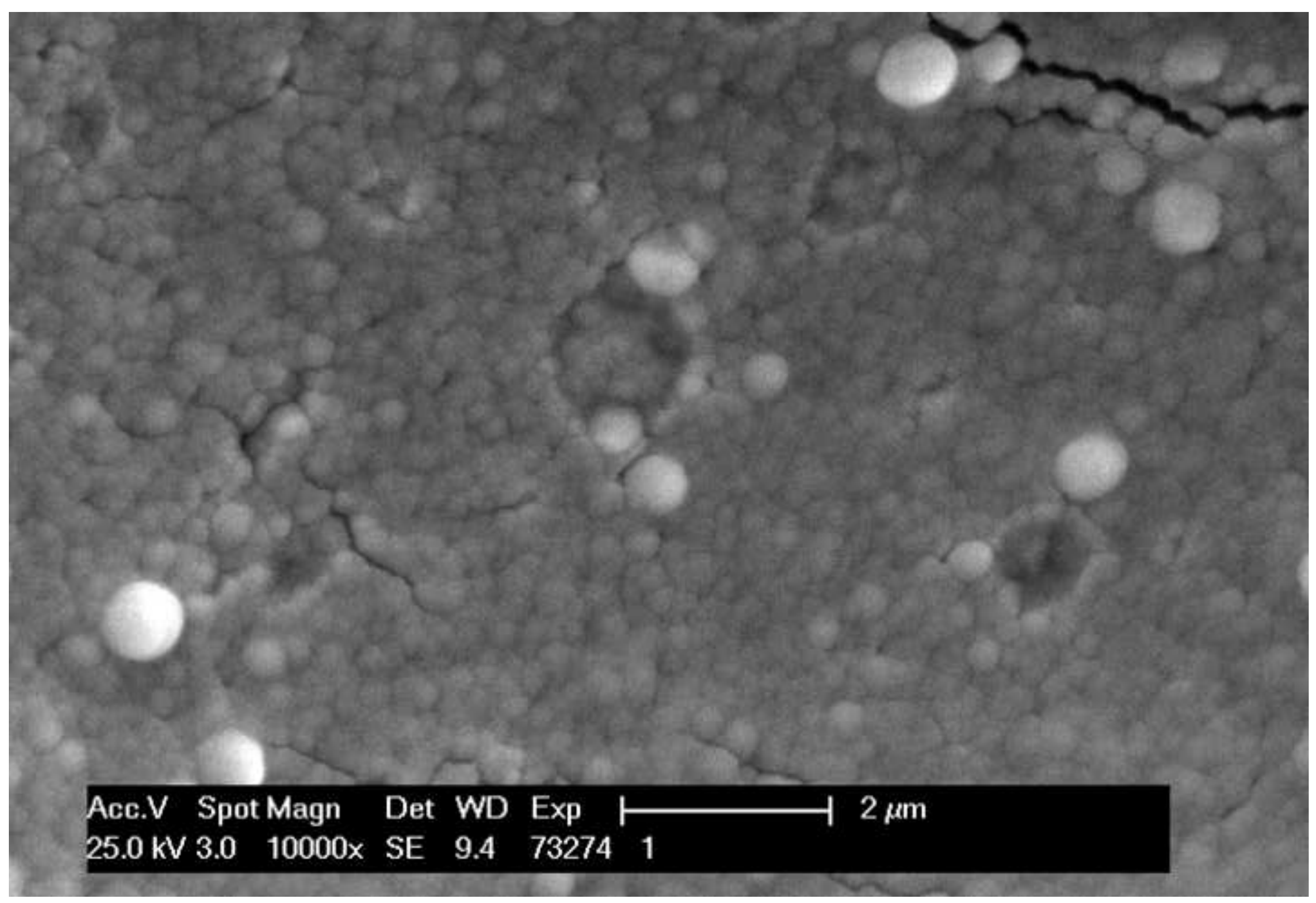

$\begin{array}{lllllll}\text { Acc.V } & \text { Spot Magn } & \text { Det } & \text { WD } & \text { Exp } & & \\ 25.0 \mathrm{kV} & 3.0 & 10000 \times & \text { SE } & 9.4 & 73274 & 1\end{array}$


Click here to download high resolution image

\section{C:\{SharedDatałKrisłFeczko|PEDMA 1.spc}

Label A: PEDMA 1

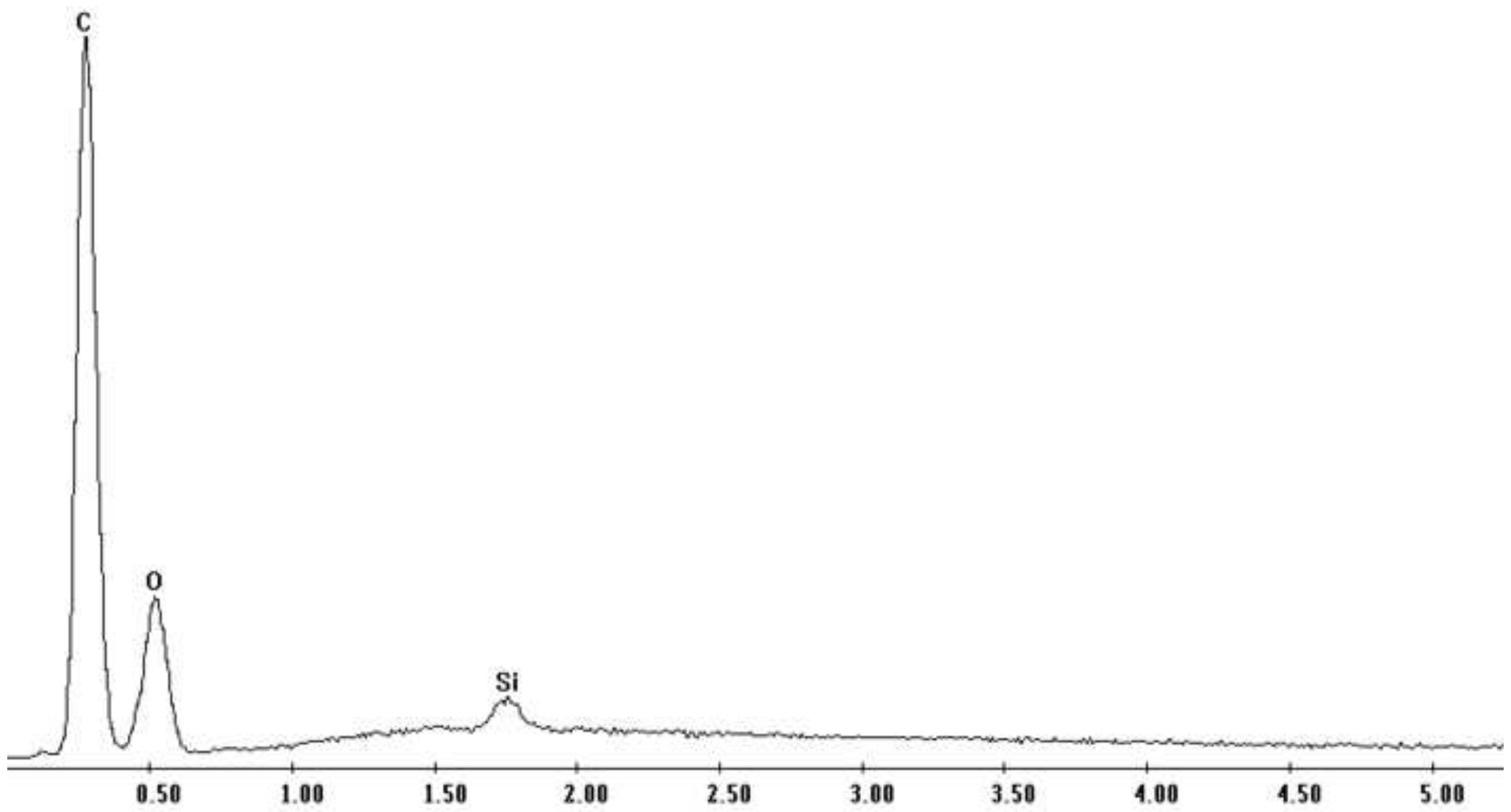




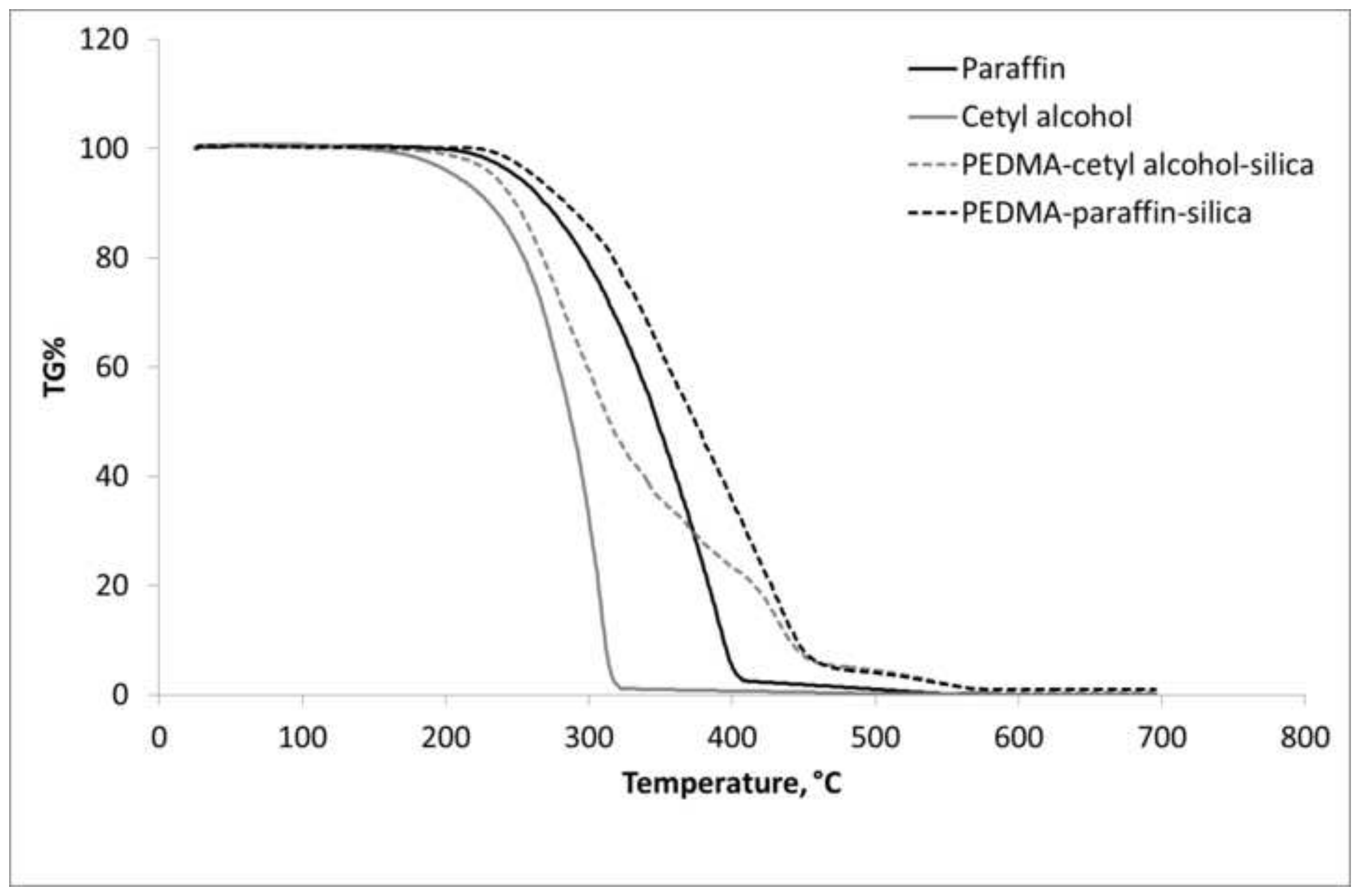




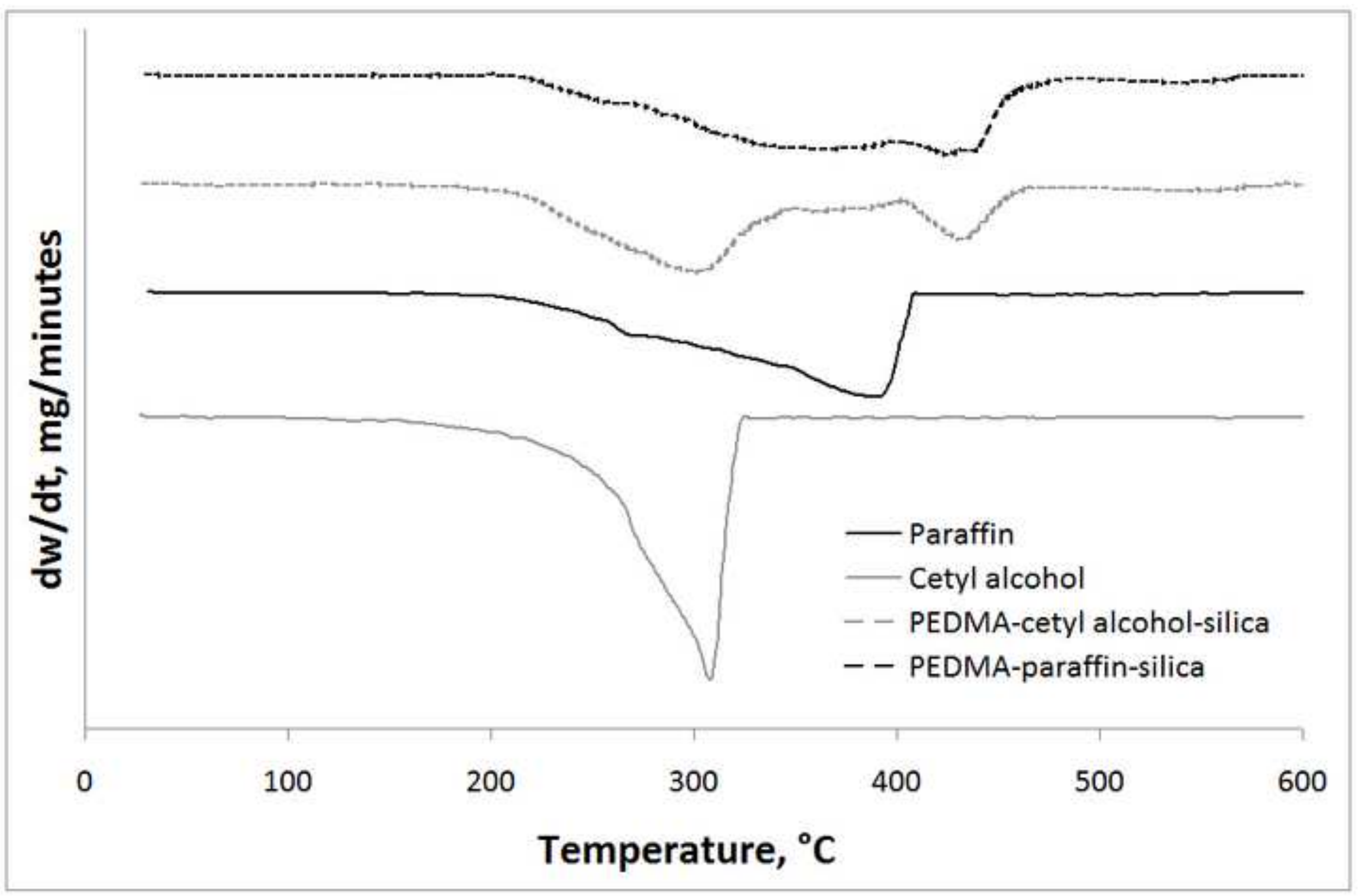


Click here to download high resolution image

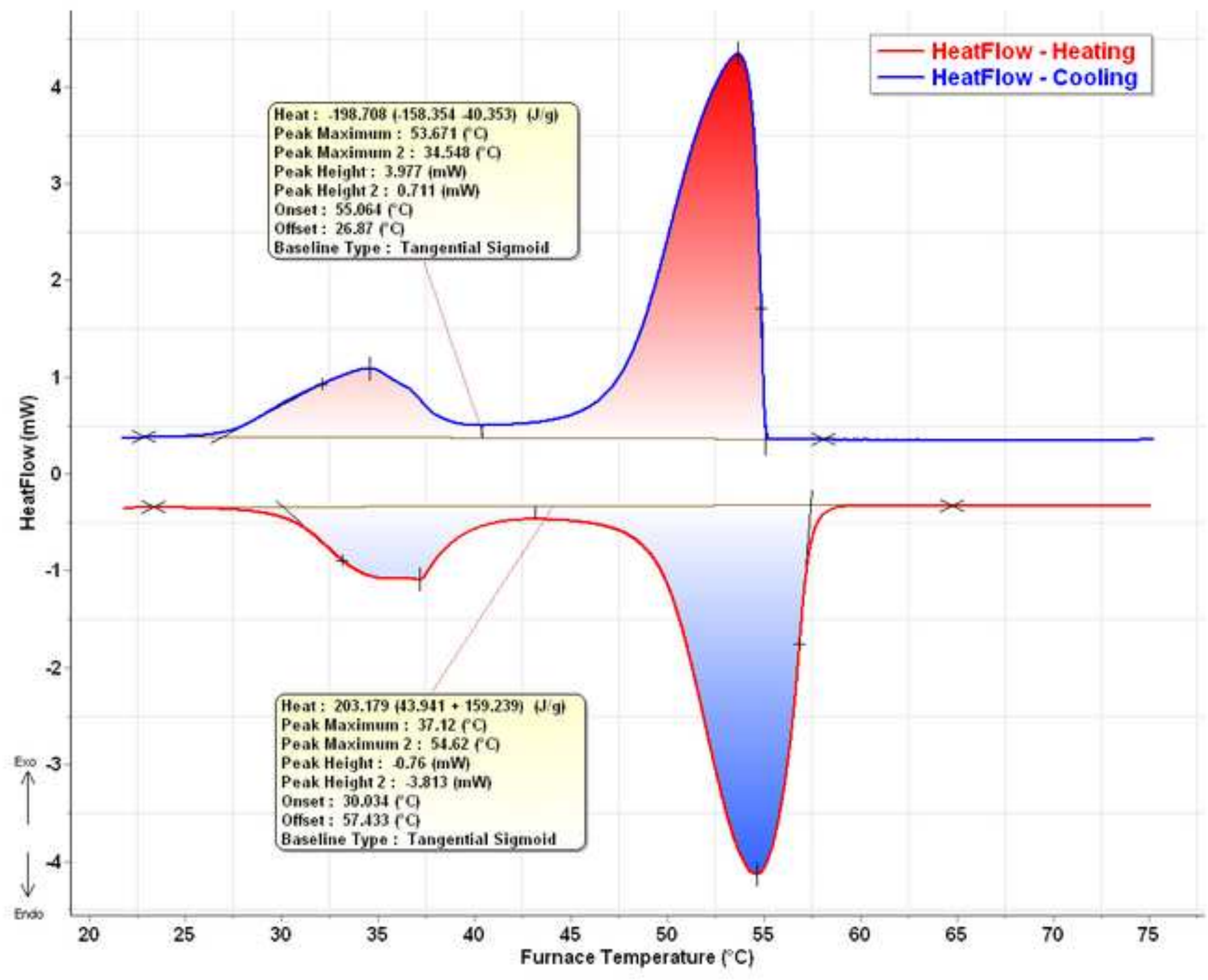


Click here to download high resolution image

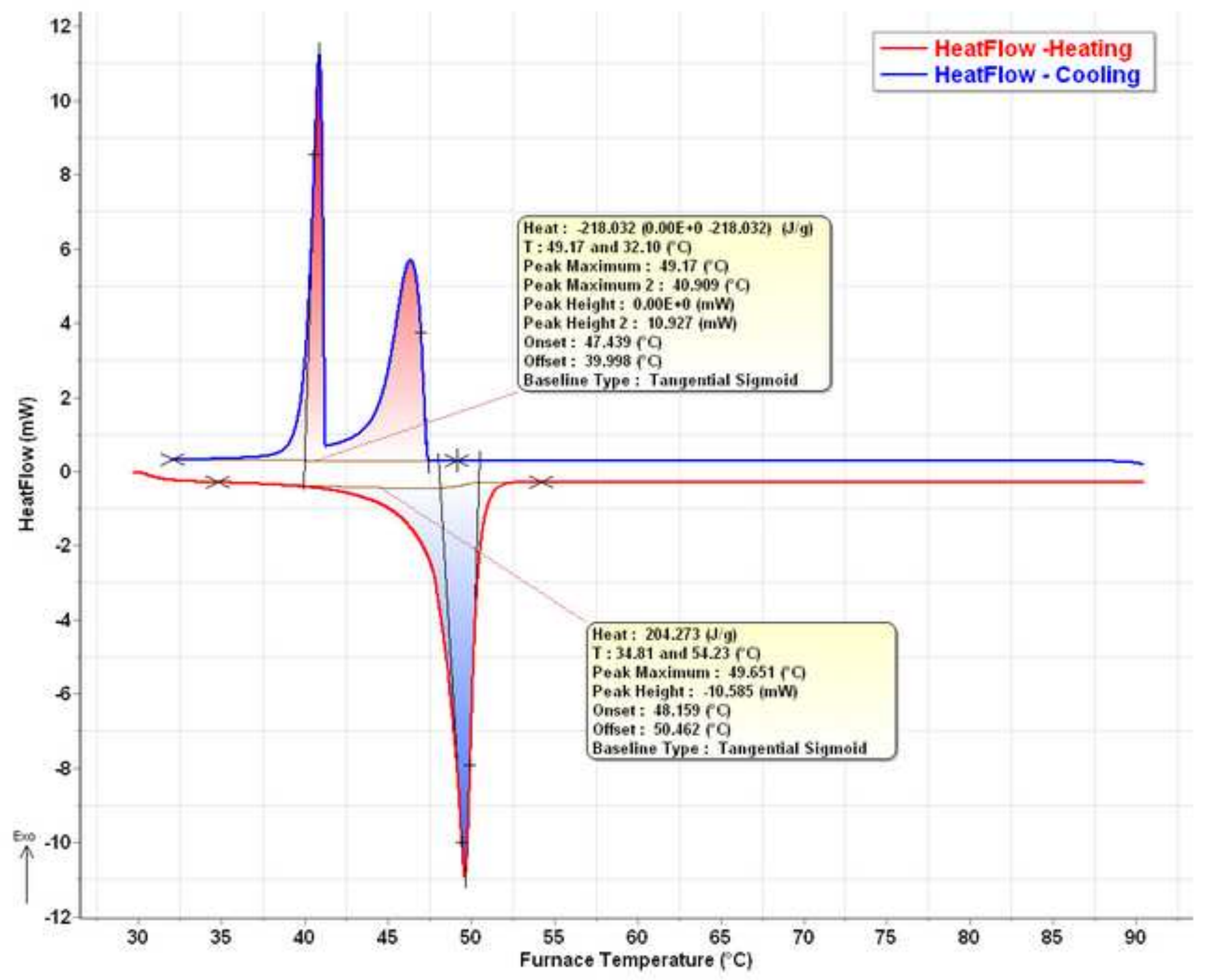




\section{Figure $7 c$}

Click here to download high resolution image

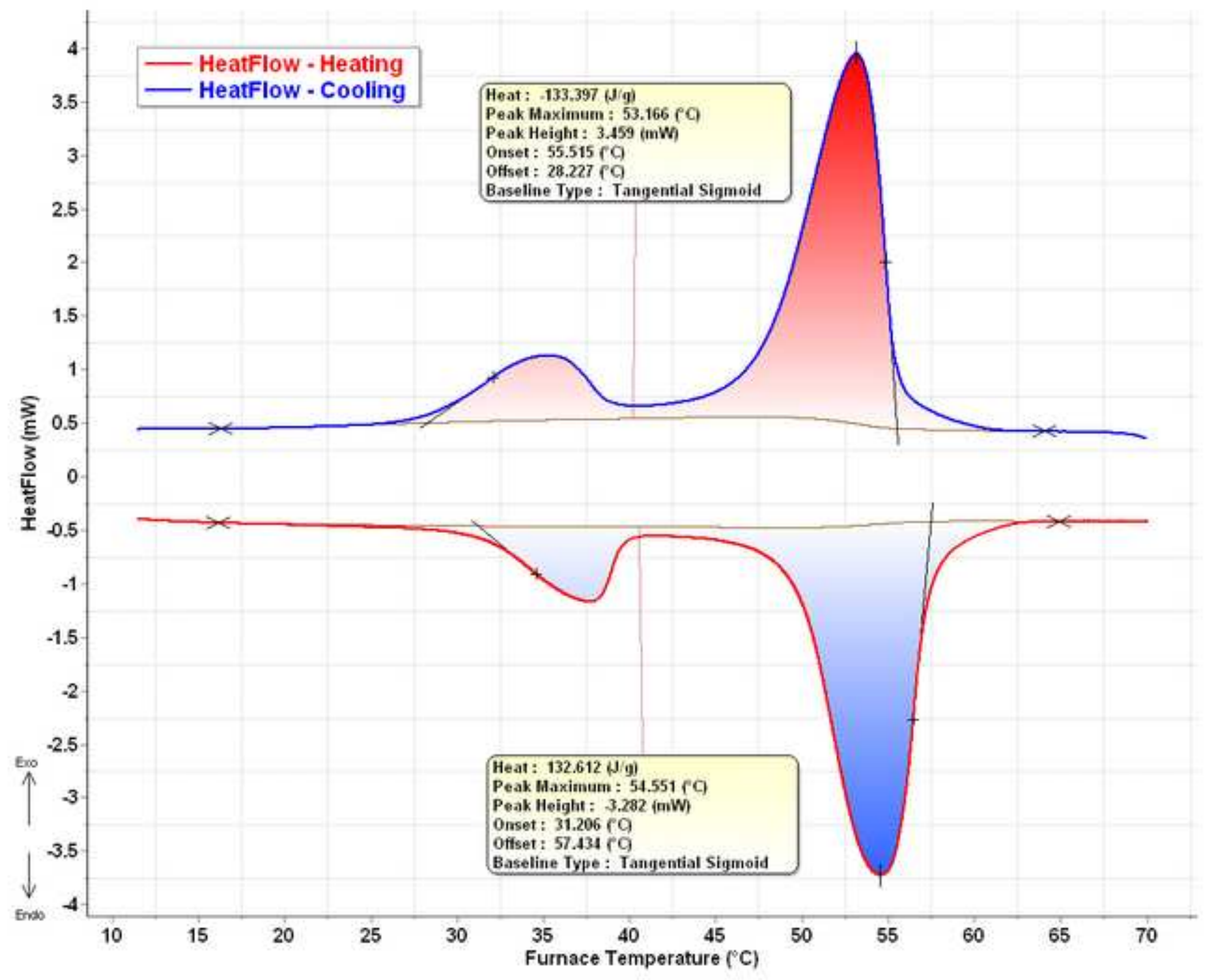


Click here to download high resolution image

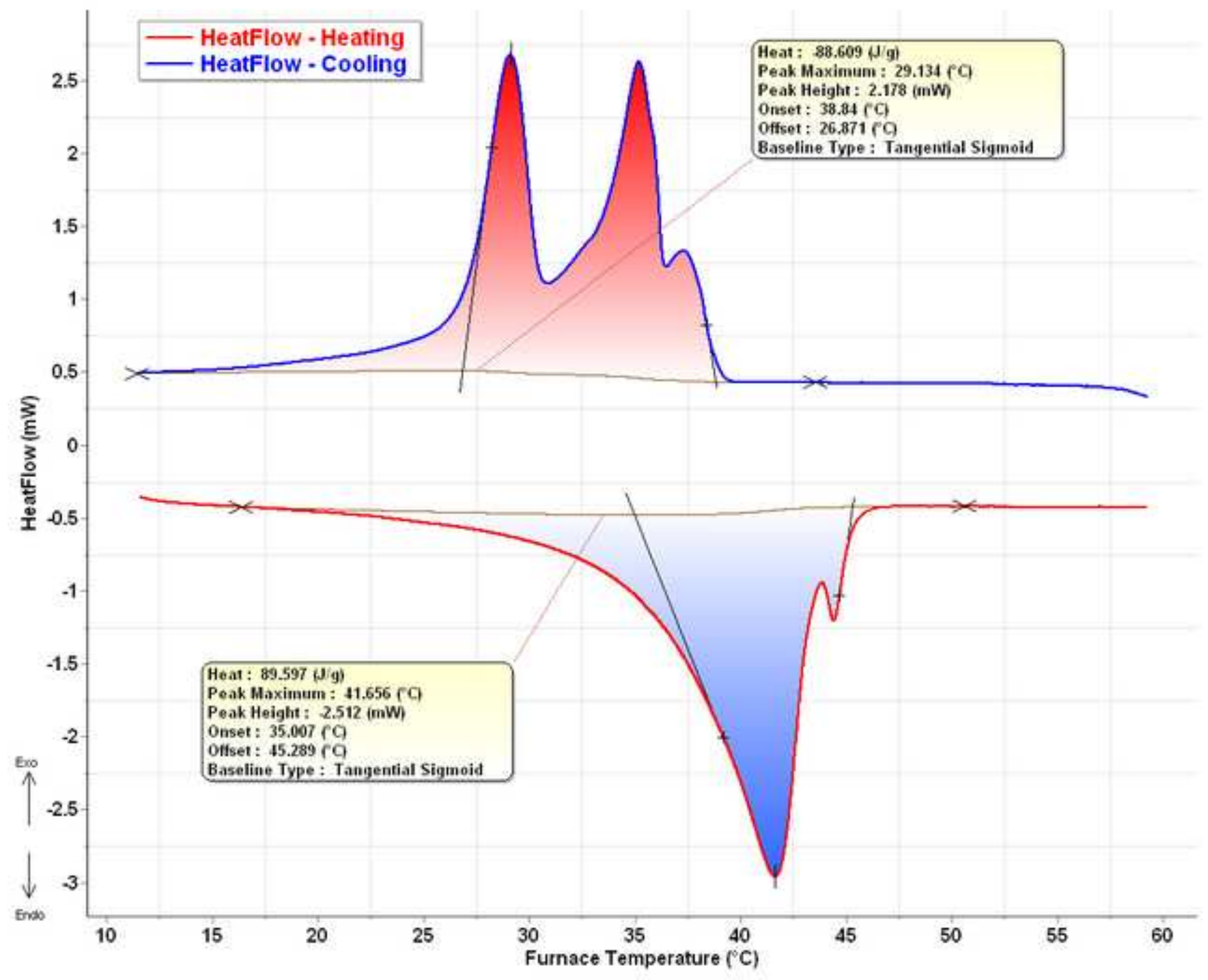


Click here to download high resolution image

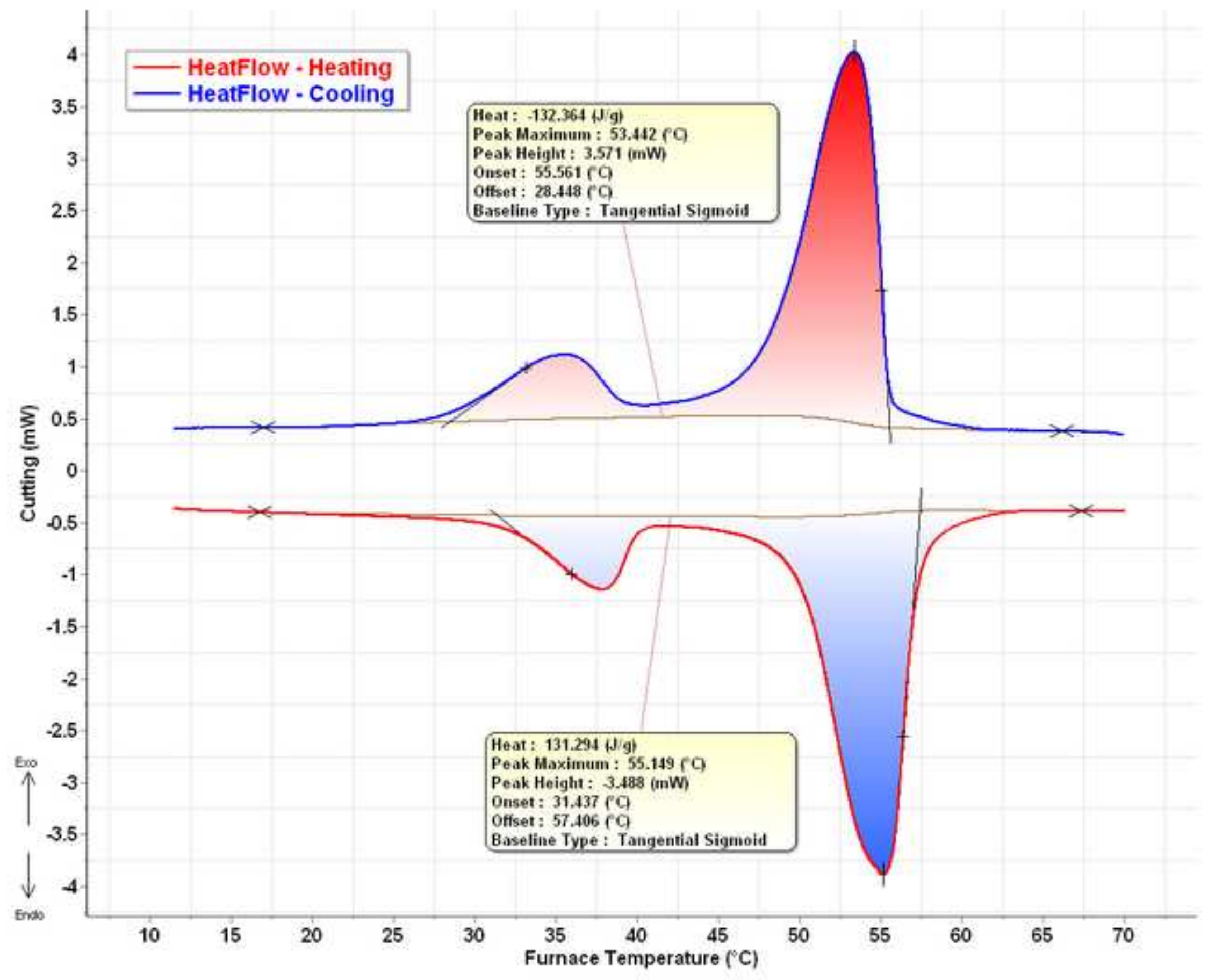


Click here to download high resolution image

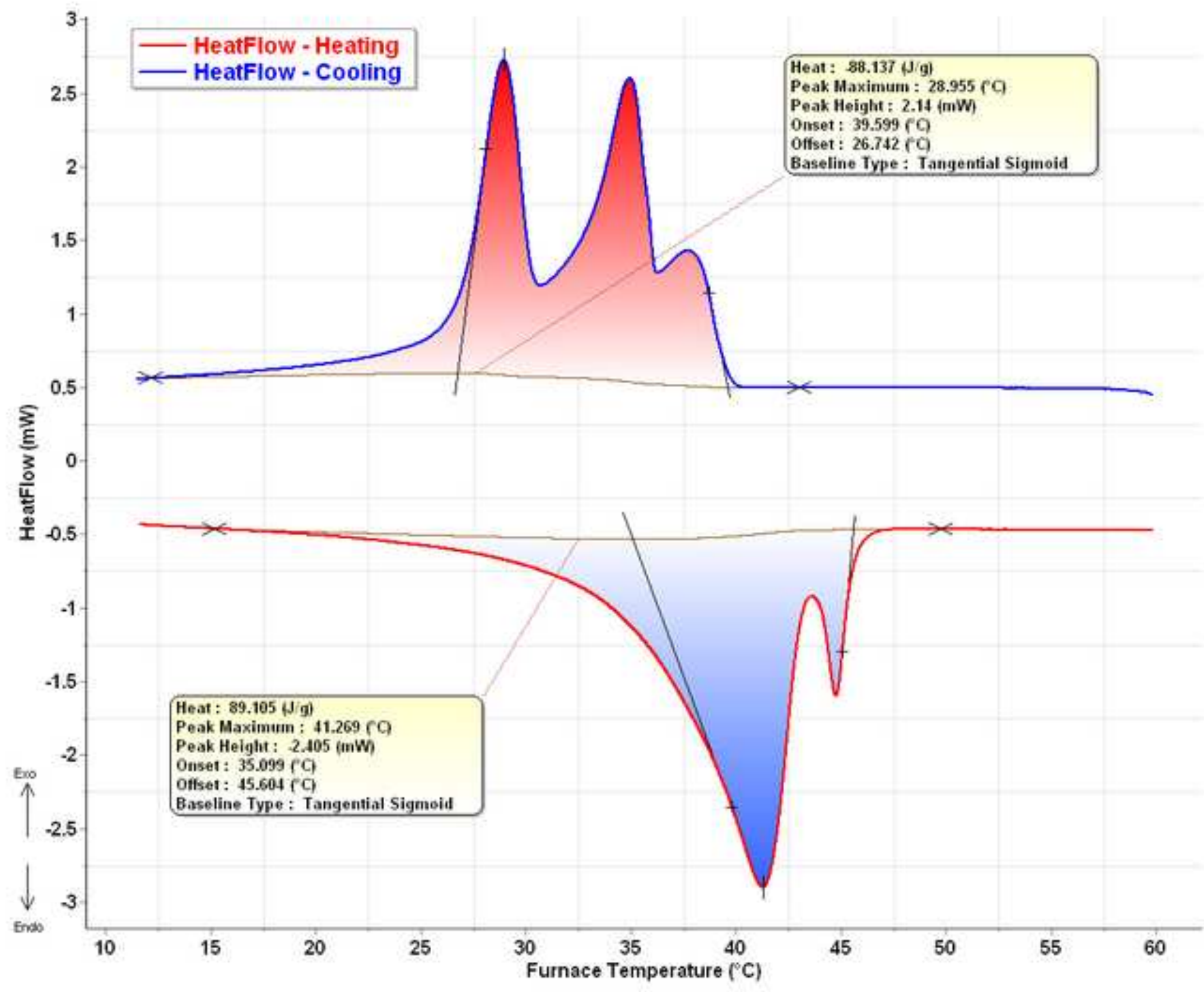


Table 1 Specific surface area $\left(\mathrm{m}^{2} / \mathrm{g}\right)$ and pore volume $\left(\mathrm{m}^{2} / \mathrm{g}\right)$ of poly(ethylene dimethacrylate) (PEDMA) beads formed for cetyl alcohol (CA) and paraffin (P) uptake.

\begin{tabular}{ccc}
\hline & $S_{\text {BET }}\left(\mathrm{m}^{2} / \mathrm{g}\right)$ & $V\left(\mathrm{~m}^{2} / \mathrm{g}\right)$ \\
\hline PEDMA (CA) & 435 & 3.0 \\
PEDMA (P) & 513 & 3.9 \\
\hline
\end{tabular}


Table 2 Volume mean diameter $(\mu \mathrm{m})$ and PCM content $(\%)$ of PEDMA beads formed for cetyl alcohol (CA) and paraffin (P) uptake.

\begin{tabular}{ccc}
\hline PCM & Volume mean diameter $(\mu \mathrm{m})$ & PCM content $(\%)$ \\
\hline CA-PEDMA & 758 & 53.1 \\
P-PEDMA & 994 & 75.6 \\
\hline
\end{tabular}

